\section{A) Check for updates}

Cite this: Analyst, 2020, 145, 3666

\title{
X-ray diffraction to probe the kinetics of ice recrystallization inhibition $\uparrow$
}

\begin{abstract}
Alice Fayter, (DD ${ }^{\text {a Steven Huband }}{ }^{\mathrm{b}}$ and Matthew I. Gibson (D) *a,c
Understanding the nucleation and growth of ice is crucial in fields ranging from infrastructure maintenance, to the environment, and to preserving biologics in the cold chain. Ice binding and antifreeze proteins are potent ice recrystallization inhibitors (IRI), and synthetic materials that mimic this function have emerged, which may find use in biotechnology. To evaluate IRI activity, optical microscopy tools are typically used to monitor ice grain size either by end-point measurements or as a function of time. However, these methods provide 2-dimensional information and image analysis is required to extract the data. Here we explore using wide angle X-ray scattering (WAXS/X-ray powder diffraction (XRD)) to interrogate 100's of ice crystals in 3-dimensions as a function of time. Due to the random organization of the ice crystals in the frozen sample, the number of orientations measured by XRD is proportional to the number of ice crystals, which can be measured as a function of time. This method was used to evaluate the activity for a panel of known IRI active compounds, and shows strong agreement with results obtained from cryo-microscopy, as well as being advantageous in that time-dependent ice growth is easily extracted. Diffraction analysis also confirmed, by comparing the obtained diffraction patterns of both ice binding and non-binding additives, that the observed hexagonal ice diffraction patterns obtained cannot be used to determine which crystal faces are being bound. This method may help in the discovery of new IRI active materials as well as enabling kinetic analysis of ice growth.
\end{abstract}

Received 25th October 2019 Accepted 16th February 2020 DOI: 10.1039/c9an02141h rsc.li/analyst est, particularly in the cryopreservation of cells and tissue, as well as proteins. ${ }^{2,11}$

Synthetic mimics of $\mathrm{AF}(\mathrm{G}) \mathrm{Ps}$ have emerged that can reproduce their properties and benefit from the scalable and tuneable synthesis of synthetic polymers. ${ }^{12-14}$ However, questions remain if these function by the same mechanisms. ${ }^{12,15}$ These include $C$-linked glycopeptides, ${ }^{16-18}$ a range of small molecules, ${ }^{3,19,20}$ polyampholytes, ${ }^{21,22}$ oxidized quasi-carbon nitride quantum dots (OQCNs), ${ }^{23}$ safranine-O, ${ }^{24}$ graphene oxide, ${ }^{25}$ zirconium acetate (ZrAc), ${ }^{26}$ nanocelluloses, ${ }^{27}$ and poly (vinyl alcohol) (PVA); which is one of the most active mimics reported to date. ${ }^{28-31}$ The development and use of appropriate assays of activity is crucial to develop this field and understand the mode of action.

Optical microscopy has been employed in the majority of experimental work concerning ice and ice-active compounds thus far. IRI activity is typically measured using either the 'splat',28,31,32 or sucrose 'sandwich', ${ }^{43}$ assays, providing visual comparisons of ice crystal wafers. Both of these assays involve nucleating small ice crystals and observing their growth over time (or more often at a fixed time point) whilst recrystallization occurs. Challenges with these microscopy techniques include time-consuming data analysis, small sampling size (micrographs of isolated regions analysed rather than whole sample) and that only $2 \mathrm{D}$ information is obtained. 
X-ray powder diffraction is used to study, in situ, the bulk of the sample. ${ }^{34,35}$ [Note, the terms WAXS and X-ray powder diffraction are often interchangeable and XRD is used here throughout]. Significant advances in technology, development of full-profile analytical methods and synchrotron radiation have aided progress in the X-ray field, as there have been vast improvements in data precision and speed of data collection. ${ }^{36}$ XRD is highly sensitive to small changes and aids identification of structural similarities and changes, especially phase or particle size changes, ${ }^{34,37}$ and has been used in many areas to monitor these changes; including observing nucleation/ growth of gold nanoparticles ${ }^{38}$ and titanium oxide particles over time, ${ }^{39}$ as well as evaluating phase structure, precipitate growth and coarsening of alloys using combined ultra-small-, small- and wide-angle X-ray scattering, ${ }^{40}$ for example. Using $\mathrm{X}$-ray techniques it is possible to report crystallographic form, polymorphism, peak intensity changes, peak splitting and peak disappearance. ${ }^{41,42}$ Scattering has been used to obtain crystal structures of cryoprotectants such as AFPs, ${ }^{43}$ though little research has been taken into their effect on ice growth. ${ }^{44}$ Studies have also monitored crystallization, polymorphism and disorder of ice upon addition of solutes such as bovine serum albumin (BSA) and sorbitol ${ }^{45,46}$ as well as investigating potential ice growth inhibition mechanisms. ${ }^{47}$ However XRD has not been used thus far to study the kinetics of ice growth, which is crucial in the design of new IRIs.

Here we report the use of XRD to probe the kinetics of ice recrystallization (and inhibition) using a range of IRI active macromolecules. The relative change in ice crystal size is determined by calculating the change in number of crystal orientations over a set time. The number of orientations is inversely proportional to the size of the ice crystals, and is obtained by integrating each individual diffraction peak as a function of $\psi$ (psi), and then plotting intensity versus $\psi$ to obtain timedependant data points proportional to the number of crystals. This is significant as it enables automated analysis of ice recrystallization (or inhibition of) with high temporal resolution, and interrogates 100's of crystals, unlike traditional optical methods. This method may help provide understanding and discovery of new IRI active materials, alongside established optical microscopy methods.

\section{Experimental section}

\section{Materials}

Poly(ethylene glycol) (PEG) (4 kDa) and poly(vinyl alcohol) (PVA) (10 kDa), phosphate-buffered saline (PBS) preformulated tablets, lysozyme and ampicillin were purchased from SigmaAldrich and used as supplied unless otherwise stated. Imidazole (Merck) and glycerol (Fisher), isopropyl- $\beta$-D-thiogalactoside (IPTG) (VWR chemical), safranine-O and phenosafranin (ACROS organics), Novex AP Chromogenic and coomassie blue stain (Expedeon) were also used as supplied. IMAC Sepharise 6 Fast Flow columns, HiLoad 16/600 Superdex 75 pg gel filtration columns and PD10 desalting columns were pur- chased from GE Healthcare and used according to manufacturer's instructions. AFGP8 CC-86-XVII was kindly provided by A. L. DeVries (University of Illinois at Urbana-Champaign, USA) and used as received. The pET20b-AFPIII plasmid encoding for a hexahistidine-tagged AFPIII from ocean pout ( $R Q A E$ isoform, M1.1HISPET20b) was kindly provided by Peter Davies (Queens University, Kingston, Canada). Competent Escherichia coli BL21(DE3) cells were sourced from New England Biolabs. Gold nanoparticles were synthesised as reported previously. ${ }^{48}$ For washing of AuNPs, Amicon Ultra-0.5 centrifugal filter units with Ultracel-30 membrane were used. $40 \mathrm{~nm}$ citrate-stabilised gold colloid solution was purchased from BBI solutions.

\section{X-ray sample preparation}

Samples were prepared, in either PBS solution or using Milli-Q ultrapure water with resistance $<18 \Omega$ obtained from a Milli- ${ }^{\odot}$ Integral Water Purification System, over a range of concentrations and $10 \mu \mathrm{L}$ was inserted into $1 \mathrm{~mm}$ thick quartz capillaries and mounted in a Linkam THMS350. Samples were cooled to $-35{ }^{\circ} \mathrm{C}$ at $30 \mathrm{C} \mathrm{min}^{-1}$, then heated to $-8{ }^{\circ} \mathrm{C}$ where $\mathrm{X}$-ray scattering was performed.

\section{XRD data analysis}

A Xenocs Xeuss 2.0 equipped with a micro-focus $\mathrm{Cu} \mathrm{K}_{\alpha}$ source and a Pilatus 300k detector was used for XRD measurements. Diffraction pattern plotting, orientation analysis and any statistical tests were performed using OriginPro 2019 software. Three major diffraction peaks: (100), (002) and (101) were focused on. Positions $(2 \theta)$ of these peaks are $22.7^{\circ}, 24.5^{\circ}$ and $25.8^{\circ}$ respectively.

\section{XRD orientation analysis}

Measurements were made repeatedly, with a counting time of 10 seconds and a 4 seconds delay between each acquisition, over 1800 seconds annealing, enabling analysis of the number of crystal orientations observed over time and comparison to 'splat' assay data. A radial integration of the 2D scattering profile was performed using FOXTROT 3.3.4 software resulting in $1 \mathrm{D}$ intensity versus $2 \theta$ plots. In addition, azimuthal integrations for each Bragg peak were performed producing 1D intensity versus $\psi$ plots. Data was then run through an inhouse MATLAB (The MathWorks, Natick, MA) programme to obtain the number of ice crystal orientations.

\section{Ice recrystallization inhibition 'splat' assay}

The sample was prepared in PBS solution, and $10 \mu \mathrm{L}$ was dropped onto a glass microscope coverslip atop an aluminum plate cooled to $-78{ }^{\circ} \mathrm{C}$ with dry ice. The thin wafer of ice that forms upon contact with the coverslip was transferred to a cryostage held at $-8{ }^{\circ} \mathrm{C}$ using liquid nitrogen, and left to anneal for 1800 seconds at this temperature. Three images were taken at different locations on this wafer at $20 \times$ zoom under cross polarizers. Using ImageJ, the numbers of crystals in the images were counted and the average crystal size per wafer was calculated as mean grain area (MGS) as well as the mean grain length size (MLGS). 


\section{Sucrose 'sandwich' ice shaping assay}

Samples dissolved in PBS buffer containing 45 wt $\%$ sucrose were sandwiched and sealed between two glass coverslips. Samples were cooled to $-50{ }^{\circ} \mathrm{C}$ and then heated to $-8{ }^{\circ} \mathrm{C}$ to anneal. The samples were then heated at $0.5{ }^{\circ} \mathrm{C} \mathrm{min}^{-1}$ until few ice crystals remained and then cooled at $0.05{ }^{\circ} \mathrm{C} \mathrm{min}^{-1}$ and the shape of ice crystals observed. Micrographs were obtained every $0.1^{\circ} \mathrm{C}$.

\section{Results and discussion}

In microscopy-based assays, smaller ice crystals (less growth) after annealing at sub-zero temperatures indicate more IRI activity, and data is reported as an area/length relative to a negative control. [Note, it is crucial to highlight the conditions for these experiments to avoid false positives. $\left.{ }^{15,49}\right]$ Fig. $1 \mathrm{~A}$ is an example micrograph from a 'splat' assay. The data is typically analysed after a set period of time, and reported as a percentage growth relative to a PBS control. This can be reported as the mean largest grain size (MLGS), where the largest crystal in view is measured from multiple samples or mean grain size (MGS), where the average size of all crystals is measured. MLGS is appealing due to its simplicity for manual measurement, but it fails to probe the whole population and a single large crystal can dominate the measurement (example shown in Fig. 1A). Fig. 1B shows a comparison of MLGS verses MGS data, which are generally in agreement, other than MGS being an area (squared) term and thus giving smaller \% value for the same amount of inhibition. The ice wafers obtained, such as in Fig. 1A, can be challenging to analyse by automated image analysis due to the irregular grain boundaries, although Ben et al. have used domain recognition software. ${ }^{50}$ Alternatively, the sucrose 'sandwich' assay, which is also used to study dynamic ice shaping (DIS); indicating ice-binding properties of a sample, Fig. 1C, is used. In this assay the crystals are more separated due to the high viscosity of the media, and image analysis can be used to identify each crystal, as shown by Budke et al. ${ }^{51}$ It is also important to be able measure growth in the media of interest, and 20-45 wt\% sucrose required for this is not relevant for all conditions, and it has emerged recently that salts have a profound effect on growth rates hence the appeal of measurement in buffers. ${ }^{52,53}$

Fig. 2A/B shows example powder diffractograms as a function of time for ice crystals annealed at $-8{ }^{\circ} \mathrm{C}$ in the presence of the potent IRI (poly(vinyl alcohol)), PVA, and a negative control (poly(ethylene glycol)), PEG. The characteristic peaks of ice at $22.7,24.2$ and $25.8^{\circ}$ (corresponding to the (100), (002) and (101) planes respectively) are clearly visible, as would be expected for hexagonal ice. ${ }^{54}$ Of note, is that in the presence of PVA, the diffractograms are constant over the whole time period, indicative of no ice recrystallization (growth). In contrast, PEG shows significant decreases in the scattering intensity for most peaks, which is consistent with ice recrystallization (larger ice crystals). This confirms that in principle, powder diffraction should allow for both end-point and kinetic analysis of IRI activity. Example ice wafers from the 'splat' assay under the same conditions are shown (Fig. 2C/D).

In this XRD analysis, no broad, low intensity peaks were observed, indicating no amorphous phases are formed in these samples upon freezing. Thus all ice observed here is polycrystalline; containing hundreds of crystallites. A percentage of these crystallites will orient to diffract the X-ray beam, thus there is a possibility the peak intensity will differ for identical samples, depending on how the crystallites are oriented. Therefore an understanding of the number of crystallites is required for evaluating the diffraction pattern. From here we will consider the number of crystal orientations as well as the observed diffraction patterns.

XRD provides details on crystallite orientations and intensity; the number of crystallite orientations for each sample, and how this number changes over continuous measurements. The sum of the intensity of the whole X-ray detector can be calculated, as well as the intensity for individual $2 \theta$ values. To enable quantitative analyses of ice recrystallization the total number of ice crystal orientations observed for a sample was calculated, which in a powder sample is proportional to the number of crystals, and hence inversely proportional to the size of the ice crystals. When ice crystals in a sample increase in size (recrystallisation), the number of grains detected by XRD decreases, which may lead to a decrease in total scattering intensity. To obtain the number of ice crystal orientations over time, the $2 \theta$ ranges that cover each of the seven peaks that are measured in the diffraction patterns are selected for each time point. An example $2 \theta$ range for the first peak is shown in Fig. 3A/B. The 2D detector image is then integrated azimuthally as a function of $\psi$ (psi) seven times with $2 \theta$ ranges determined from the diffraction peaks. The step size in $\psi$ for this was $0.2^{\circ}$. The size of the detector limits the available orientation range in $\psi$ to between 76 and $105^{\circ}$. The intensity versus $\psi$ data for the selected $2 \theta$ range is plotted and the number of data points above a determined background value $(1.5$ counts used) are then counted, these data points refer to the number of orientations and are filled in red to show an example of IRI active and inactive samples in Fig. 3C/D. As the concentration of an IRI active substance increases, the relative ice growth rate is expected to decrease due to the inhibition of ice crystal growth. This is because there are many orientations, which correspond to many small crystals. If there is an observed decrease in the number of orientations, this is due to Ostwald ripening; where the larger crystals increase in size at the expense of the small crystals.

To evaluate the use of this method, a range of known IRI active compounds (biological and synthetic) were tested and compared against the standard 'splat' assay, Fig. 4. In each case, XRD experiments were performed over 1800 seconds (30 min). Fig. 4A shows PVA, with the MLGS values trending with the inverse number of orientations. Fig. 4B shows PEG (a negative control), indicating essentially no activity even at very high concentrations (up to $50 \mathrm{mg} \mathrm{mL}{ }^{-1}$ ), where non-specific effects dominate. Encouraged by these results, the self-assembling IRI active safranine-O was tested, verses phenosafranin, 

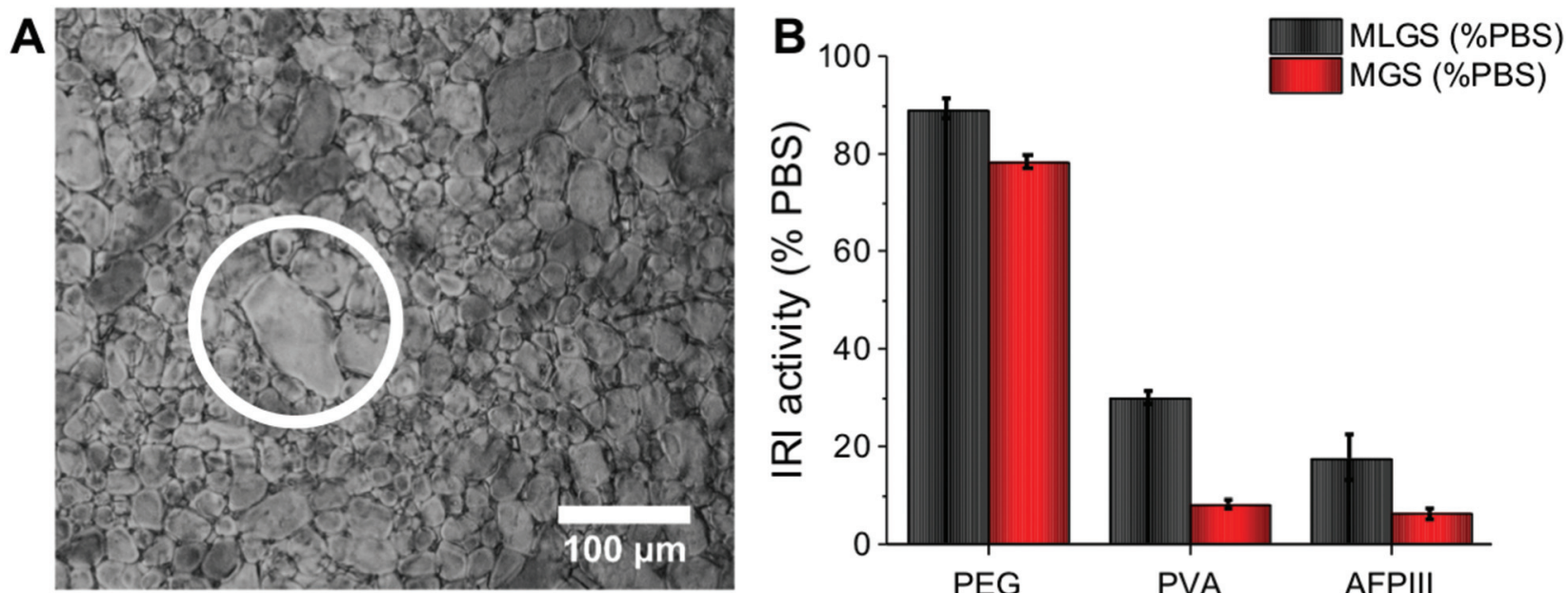

C
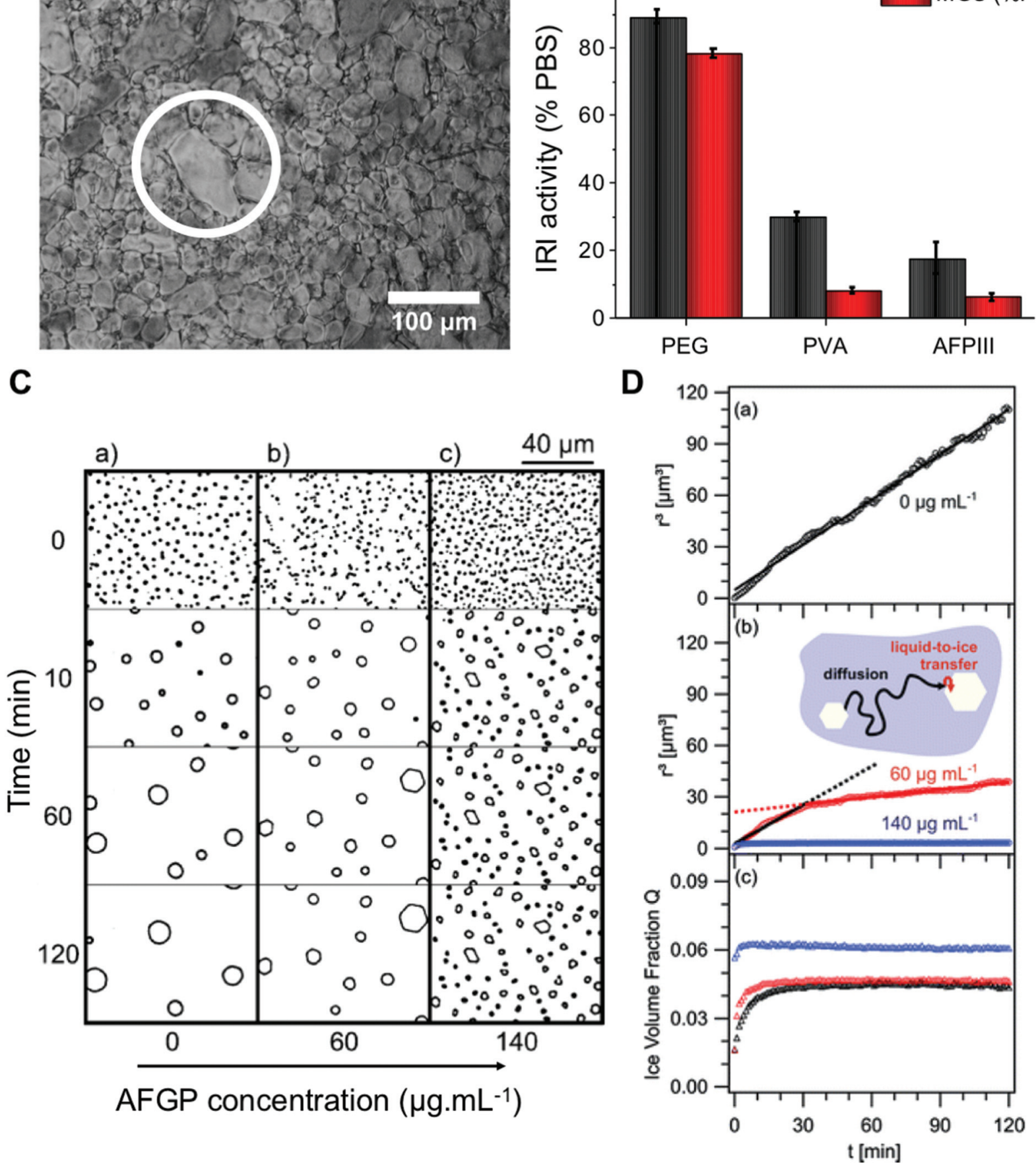

Fig. 1 Optical microscopy analysis of ice recrystallization. (A) Example ice wafer containing $1 \mathrm{mg} \mathrm{mL}^{-1} 10 \mathrm{kDa}$ PVA in PBS after 30 minutes. An example larger grain (white circle) is shown, which skews results when only 'largest grain size' is considered; (B) comparison of MLGS and MGS for some IRI active compounds. Concentrations $=1 \mathrm{mg} \mathrm{mL}^{-1}$. Errors are standard deviation; (C) micrographs of ice crystals grown in $45 \mathrm{wt} \%$ sucrose: (a) control solution, (b) $60 \mu \mathrm{g} \mathrm{mL}{ }^{-1} \mathrm{AFGP}$, (c) $140 \mu \mathrm{g} \mathrm{mL} \mathrm{L}^{-1} \mathrm{AFGP}$; (D) data obtained through calculating mean ice crystal radii, $r^{3}$, in $45 \mathrm{wt} \%$ sucrose solutions from the micrographs, (a) control solution, (b) $r^{3}$ in solution containing 60 and $140 \mu \mathrm{g} \mathrm{mL}^{-1} \mathrm{AFGP}$, and their associated fits (solid lines), (c) temporal development of ice crystal volume fraction, Q, of data from (a) and (b). Reprinted (adapted) from with permission from Koop et al., ${ }^{51} \mathrm{~J}$. Phys. Chem. B, 2009, 113(9), 2865-2873. Copyright (2009) American Chemical Society. All data obtained at $-8^{\circ} \mathrm{C}$. 

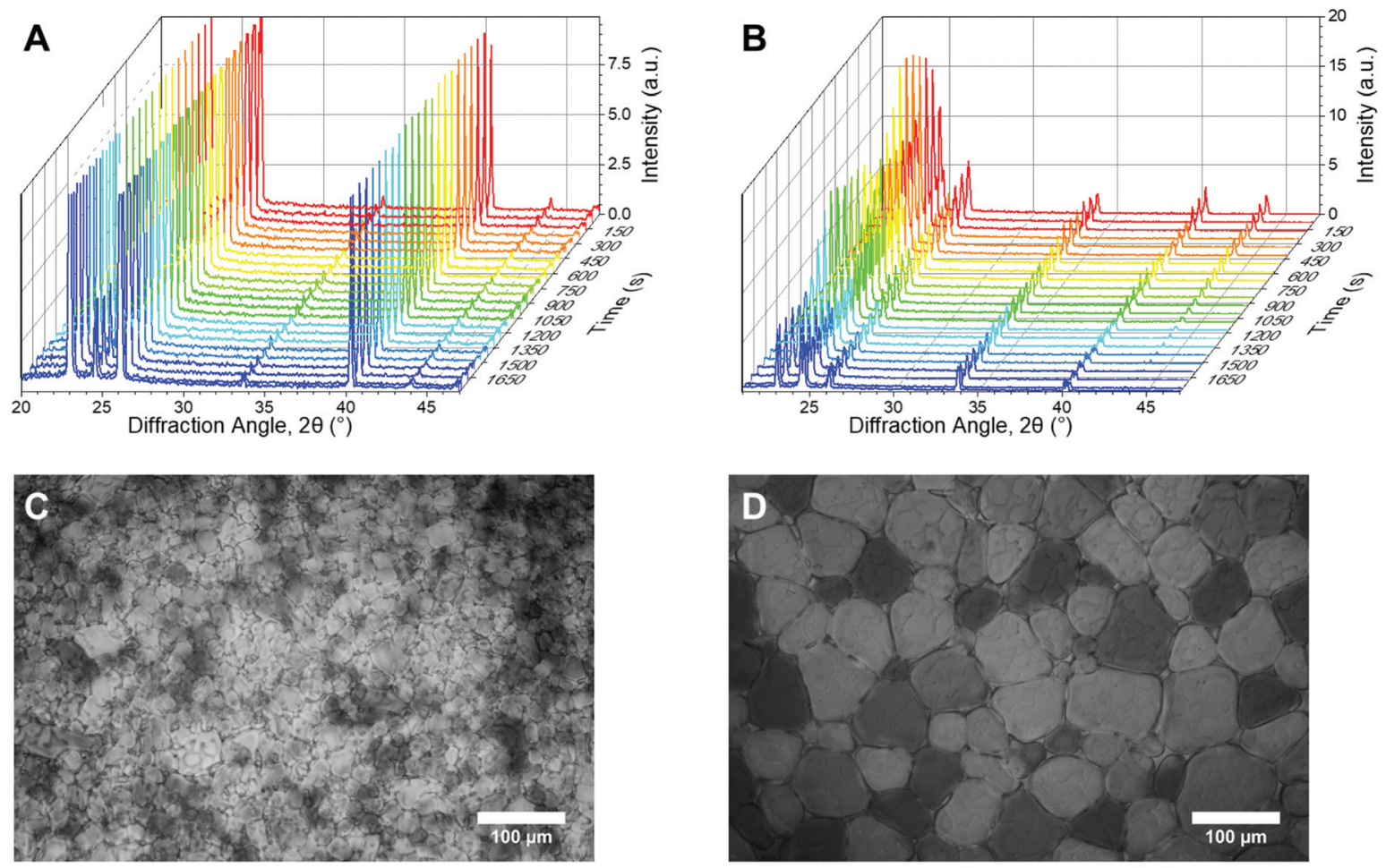

Fig. 2 Example XRD and microscopy data. (A and B) Waterfall plots of diffraction patterns used for kinetic analysis of change in number of crystal orientations, obtained using powder diffraction, over time for (A) PVA and (B) PEG; (C and D) Example micrographs for (C) PVA and (D) PEG. All data obtained at $-8^{\circ} \mathrm{C}$. [Polymer] $=3 \mathrm{mg} \mathrm{mL}^{-1}$.
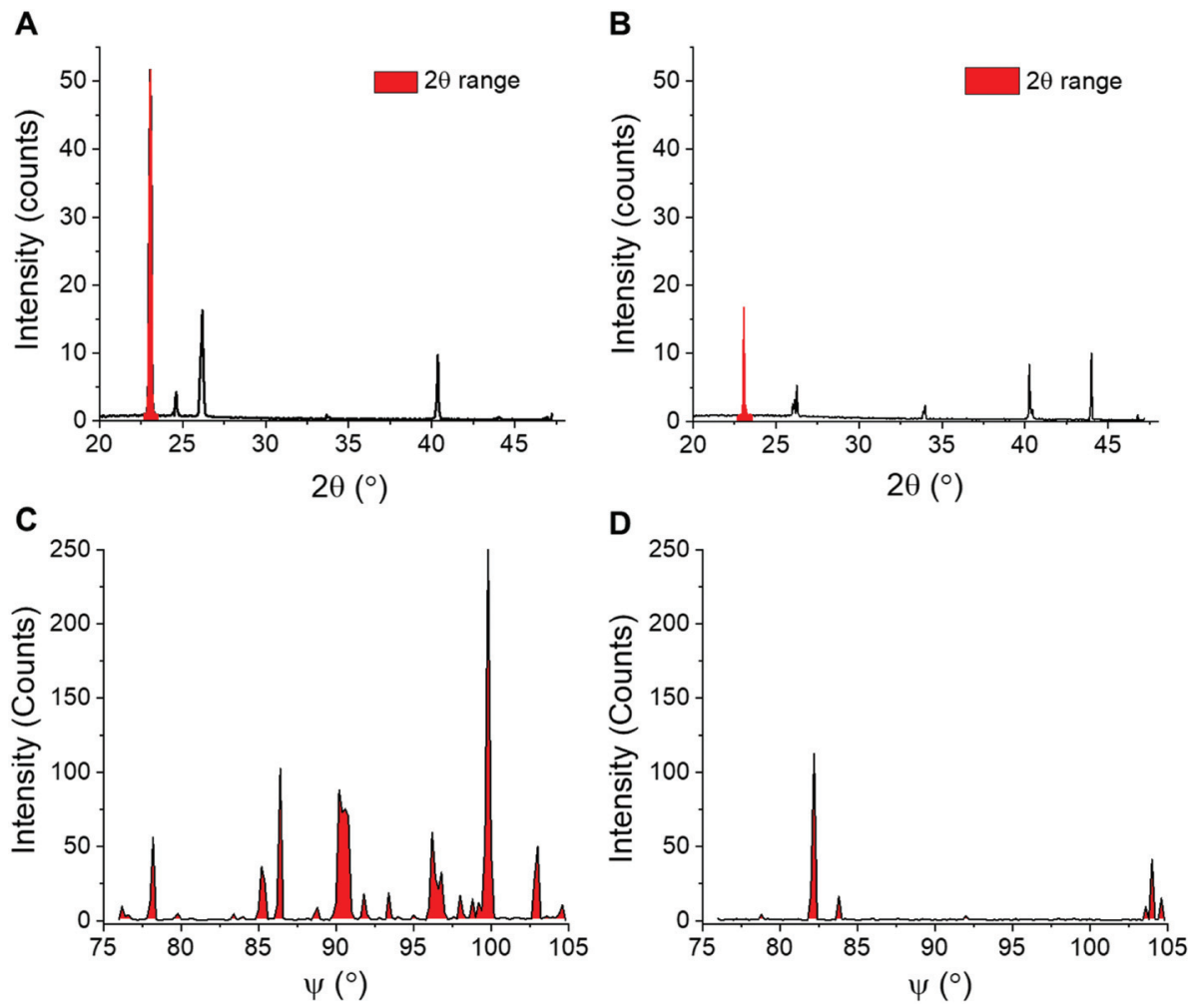

Fig. 3 Extraction of ice recrystallization data. (A and B) Example XRD plots used to calculate crystallite orientations of (A) IRI active PVA and (B) nonIRI active PEG, plotted as a function of $2 \theta$. Selected $2 \theta$ ranges used to analyse the crystallite orientations scattering are coloured red; (C and D) Orientation plots of (C) PVA and (D) PEG, plotted with as a function of $\psi$ for intensities measured in the selected $2 \theta$ range. 
A

c

E
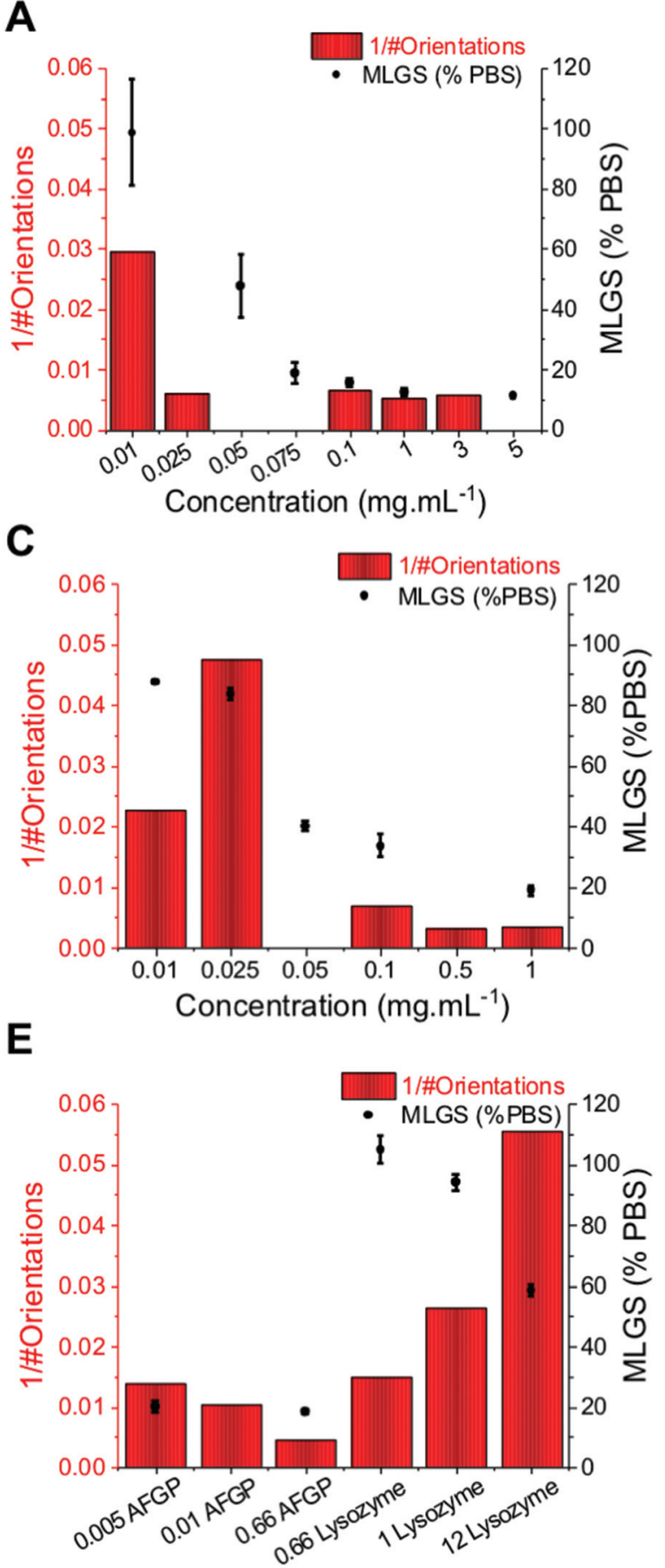

B

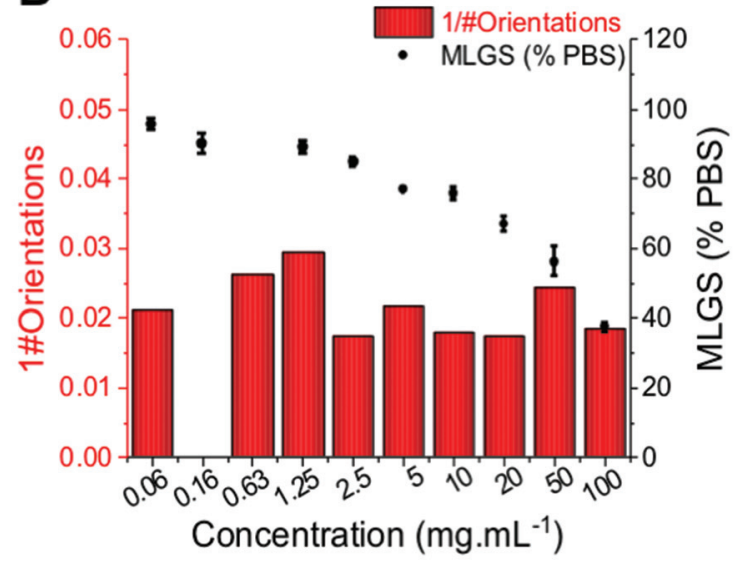

D

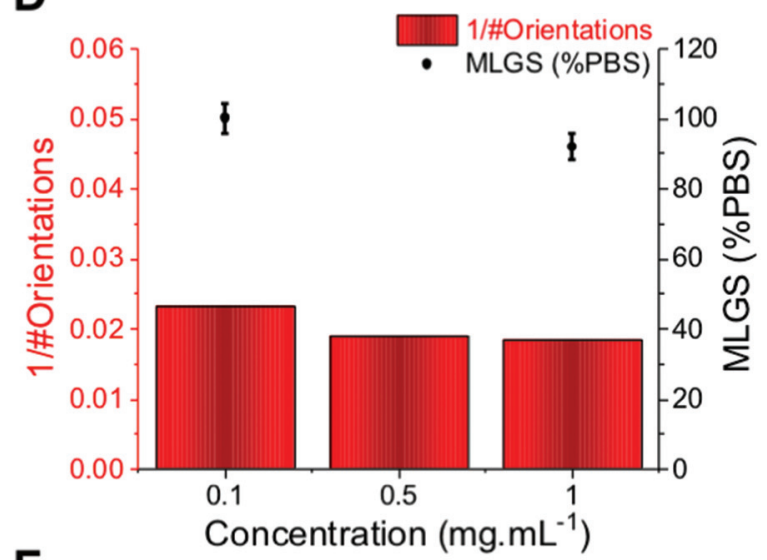

F

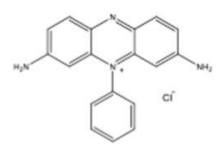

Phenosafranin<smiles>CC(C)(C)C(O)C(C)(C)C</smiles>

PVA<smiles>CCOCCC(C)(C)O</smiles>

Safranine-O
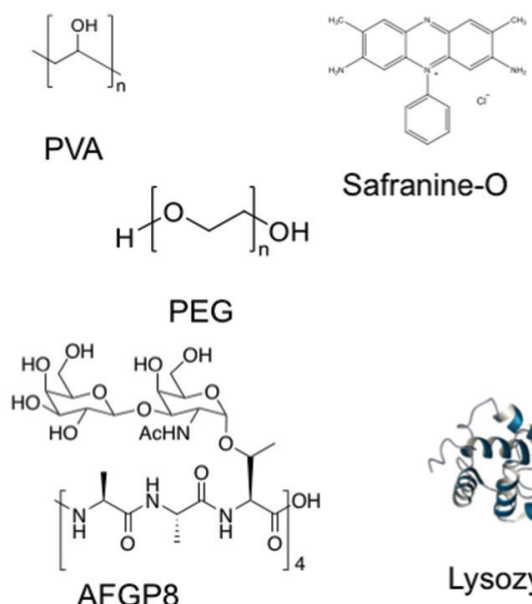

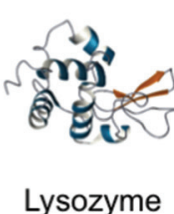

Fig. 4 Comparison of orientation analysis (here shown as inverse number of observed ice crystal orientations after 1800 seconds) and IRI activity (shown as mean largest grain size, MLGS, as a percentage of PBS) over a range of concentrations for all molecules used here; (A) PVA; (B) PEG; (C) safranine-O; (D) phenosafranin; (E) AFGP compared to lysozyme; (F) Structures of all used. All concentrations are in $\mathrm{mg} \mathrm{mL}^{-1}$. All data obtained at $-8{ }^{\circ} \mathrm{C}$. Errors are standard deviation from at least 3 individual repeats. NB. Not all concentrations of inhibitors were tested using each assay.

which cannot self-assemble and has no IRI. ${ }^{24}$ By both MLGS and XRD analysis, it can be seen that only safranine-O inhibits ice growth. Fig. 4E, shows a comparison of AFGP8 (antifreeze glycoprotein from Dissostichus mawsoni, $2.6 \mathrm{kDa}$ ) which is one of the most potent IRI's known, ${ }^{15}$ and lysozyme as a negative protein control. Again, the MGLS and XRD methods show broad agreement, demonstrating that this is a valid analysis method for IRI activity and is complementary to the traditional optical based methods.

A common challenge in the microscopy-based methods is extracting kinetic information. For XRD this is far simpler; the Pilatus $300 \mathrm{~K}$ detector system used here enables 3D data collec- 
tion every 14 seconds. This diffraction data includes, for example, information on number of crystal orientations for all measurements, which can be analysed to give kinetic information. Fig. 5A shows the change in the number of orientations (i.e. total number of crystals) for ice in the presence of
non-IRI active PEG. There is a clear decrease in number of crystals due to ice growth over the time course of the experiment. The same analysis for PVA shows a constant number of orientations, indicating a static process, and hence recrystallization is fully inhibited. It is important to note at this point that the
A

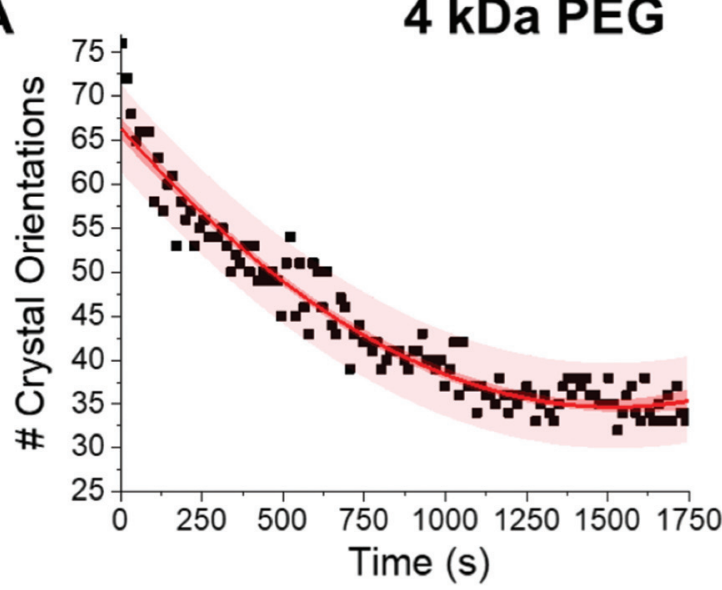

C<smiles>OCCOCC1CCCCC1</smiles>

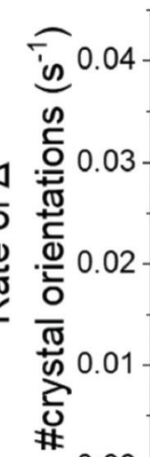
0.00

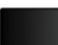

$E$ PVA

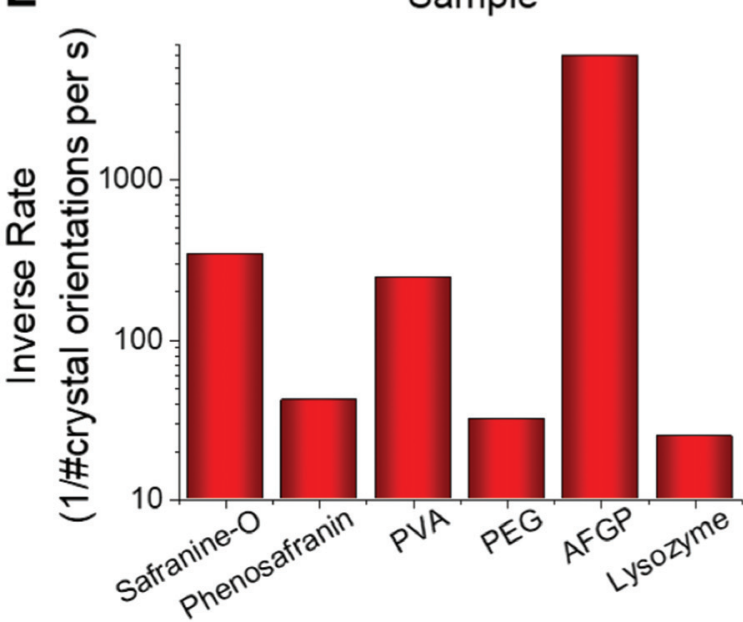

B
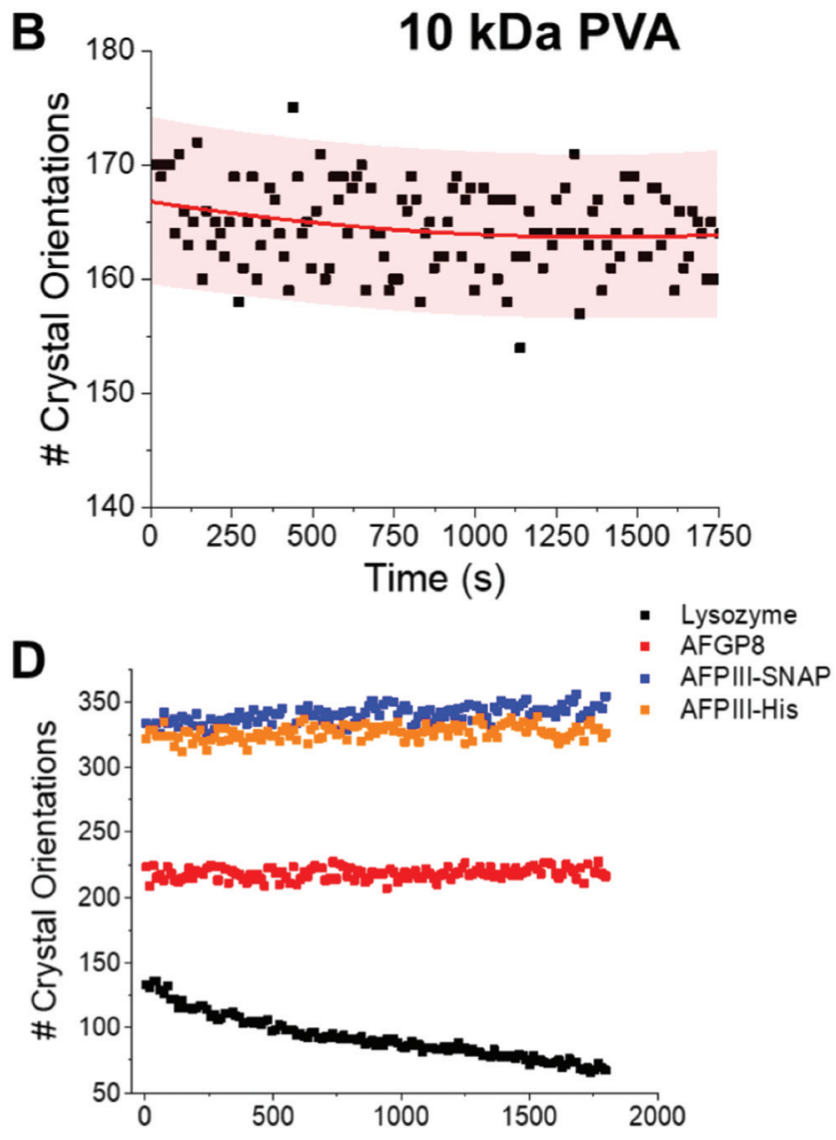

$\mathbf{F}$

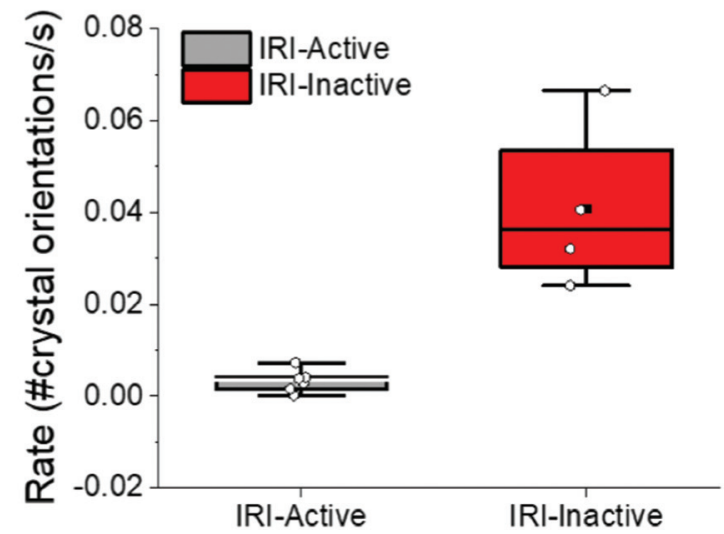

Fig. 5 IRI analysis by XRD. Kinetic profiles for (A) PEG $\left(3 \mathrm{mg} \mathrm{mL}^{-1}\right)$ and (B) PVA $\left(3 \mathrm{mg} \mathrm{mL}^{-1}\right)$ at $-8^{\circ} \mathrm{C}$; (C) comparison of PVA/PEG rates of ice growth from A/B. [Polymer] $=3 \mathrm{mg} \mathrm{mL}^{-1}$; (D) number of crystal orientations as a function of time for a range of proteins. [Lysozyme] $=0.7 \mathrm{mg} \mathrm{mL}^{-1}$, [AFPIII-His] $=0.4 \mathrm{mg} \mathrm{m}^{-1}$, [AFPIII-SNAP] $=0.1 \mathrm{mg} \mathrm{mL}^{-1}$, [AFGP8] $=0.07 \mathrm{mg} \mathrm{mL}^{-1}$; (E) inverse average rate of change in number of observed ice crystal orientations over 1800 seconds for a small molecule (safranine-O), polymer (PVA) and protein (AFGP) compared to their relative IRI inactive controls (phenosafranin, PEG and lysozyme); (F) box plot of the mean gradients of change and standard deviation for IRI active (grey) and inactive samples $\left(\right.$ red). Mean $=$ black square, individual data points $=$ white hexagons. All data obtained at $-8^{\circ} \mathrm{C}$. 
actual magnitude of the number of crystals will vary in each sample due to the different nucleation rates (which is also hard to control), however the change is the part which is of interest here, and data can be normalised to the $t=0$ points.

Relative ice crystal growth rates were obtained from the gradient of change in number of crystal orientations, examples shown in Fig. 5, showing that a parameter for ice growth can be extracted if required. The steeper the slope, the faster the ice crystal growth. The rate of change in number of crystal orientations is much greater for PEG than for PVA. Fig. 5D shows this analysis for several other compounds also, confirming the trends. For example, the rate of change in ice crystal orientations for AFPs are slightly faster than that of AFGP8, this may be due to their slightly lower IRI activity, (see ESI $\dagger$ ). Further measurements on more complex structures have been performed, which exhibit the same trends, showing that this
XRD method works for a range of structures, Fig. S1.† However they are still much lower rates than that of lysozyme. The rates of crystal growth for IRI active molecules tested can be seen to be close to zero, whereas that of the inactive controls it is higher, Fig. 5E/F.

IRI is not the only macroscopic property of ice binding/antifreeze proteins. Ice shaping, due to binding to specific crystal faces also occurs ${ }^{12,13}$ although it has emerged that binding might not be essential for IRI and hence the two properties are not always present. ${ }^{3,12}$ Fig. 6 shows ice diffraction patterns and ice shaping for all three groups of compounds tested here. The difference in the diffraction patterns between water and PBS was first analysed to confirm similarities and differences - this was due to the salts' potential colligative effects on ice growth and hexagonal ice (Ih) structure, Fig. 6A/B. The results from PBS samples are more relevant to biological samples and
A

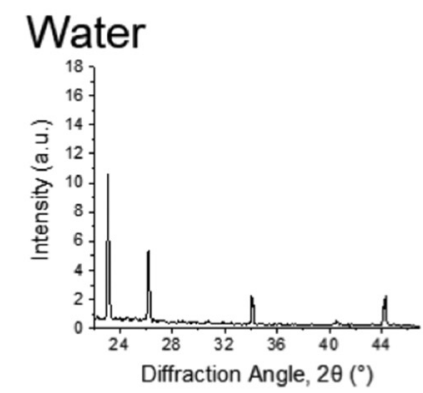

C

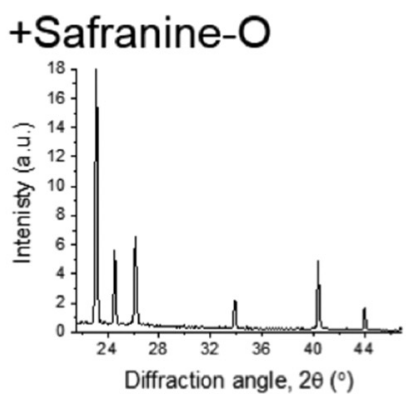

$\mathbf{E}$
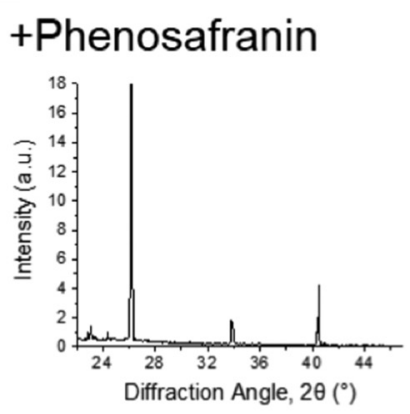

\section{B}
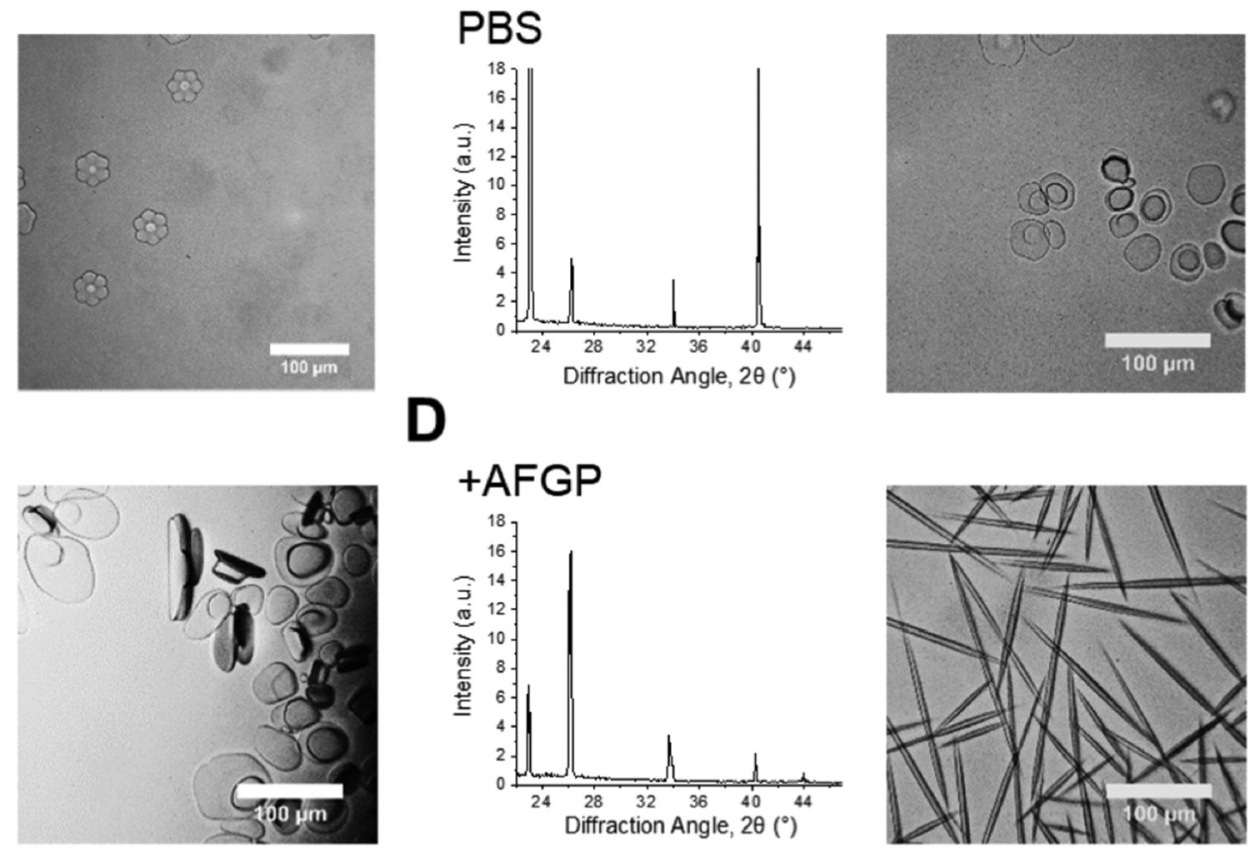

D
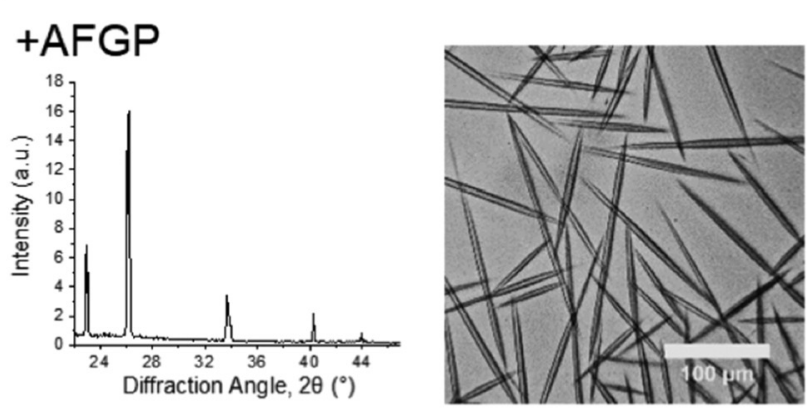

$\mathbf{F}$

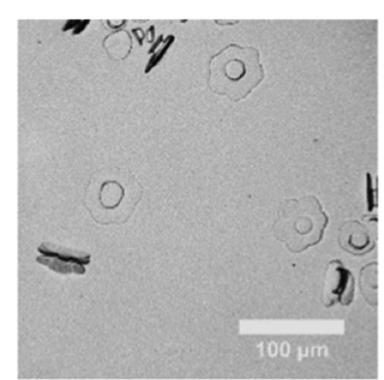

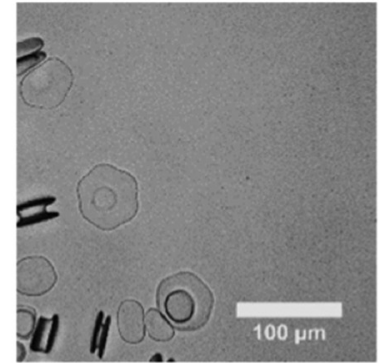

Fig. 6 Crystal habit modifications by additives. Left: Ice diffraction patterns after $1800 \mathrm{~s}$ annealing for: samples with known ice binding and shaping abilities, their relative non-binding controls and water/PBS. Right: Optical microscopy ice morphology analysis in 45 wt\% sucrose of samples in the corresponding diffractograms. Scale bars $=100 \mu \mathrm{m}$. [Safranine-O] $=1.0 \mathrm{mg} \mathrm{mL}^{-1}$; [AFGP8] $=3.0 \mathrm{mg} \mathrm{mL}^{-1}$; [phenosafranin] $=1.0 \mathrm{mg} \mathrm{mL}$; [lysozyme] $=1.0 \mathrm{mg} \mathrm{mL}^{-1}$. 
those in water would be more relatable to samples used in other fields, for example engineering and atmospheric chemistry. The structure for both is deemed to be Ih, though there are slight intensity variations for the Ih pattern between the two, but intensity variations are also seen in other samples, and are due to which grains orient towards the beam and not related to any 'ice-activity' of PBS.

Sometimes the $(002)$ plane $\left(2 \theta=24.2^{\circ}\right)$ is said to be missing in ice samples with additives ${ }^{55,56}$ and is often explained as the additive showing ice binding/DIS activity. Here measurements are taken using a static capillary, where the ice grains formed are too large to ensure a fully random distribution is sampled, thus structure analysis cannot be completely determined. A potential method to solve this problem would be to perform XRD whilst spinning the capillary, however there could still be preferred orientation effects. Due to differences in experimental set-up it is difficult to determine whether previous studies had randomly oriented grains/sampled a large enough range of grains to confirm ice binding/DIS. ${ }^{47,56,57}$ Measurements made by freezing on a surface (i.e. flat), in particular, will be strongly affected by preferred orientation and thus may not give clear evidence of binding. Hence, the disappearance of a peak cannot solely be used to describe ice crystal morphology/binding accurately. For example, AFGP affects ice morphology by binding the primary prism plane, so would have a peak missing corresponding to this plane, but pure water (or PBS) would show all Ih peaks, however the $24.2^{\circ}$ peak is missing in these samples, Fig. 6A/B/D. The same peak should also be missing for safranine-O, however is observed here, Fig. 6C. Similarly, phenosafranin and lysozyme have no known ice-binding so the lack of $24.2^{\circ}$ peak seen in Fig. 6E/F cannot represent this. This shaping or lack of ice shaping is observed in the provided micrographs. When there is a change in ice grain size, particularly growth due to Ostwald ripening, fewer crystal orientations are observed and peak intensity changes. This can cause particular orientations to not scatter the X-ray beam leading to peak disappearance, thus the diffraction pattern peaks cannot be used to describe ice crystal morphology accurately in these disordered samples. This is crucial to show the limitations and applications of this method to explore the kinetics of ice recrystallization as a complementary tool to optical microscopy.

\section{Conclusions}

Here, X-ray powder diffraction is used as a non-invasive method for 3D kinetic analysis of ice crystal growth (recrystallization) and compared to the more commonly used optical techniques. This method is complementary as measurements are performed in a capillary (3D conditions), which facilitates the measurement of a larger number of crystals in the system, as well as being preferable to $2 \mathrm{D}$ systems, where the sample holder will have a greater effect on the growth mechanisms. Furthermore, data can be obtained every 14 seconds (in this particular instrument, with the rate depending on individual facilities), enabling real-time kinetic analysis without analysing each frame from videos. Ice growth was followed by calculating the relative number of ice crystal orientations from $2 \theta$ ranges that cover each of the seven Ih peaks from each diffraction pattern at every time point. We developed a fitting method to obtain kinetic data, which consisted of plotting the number of crystal orientations as a function of time and obtaining the gradient of change in number of orientations. This was then extracted as a rate and compared to that of pure water and PBS controls. Using this method we compared the IRI activity of a range of synthetic and biological IRI's and found that it was in good agreement optical methods with the advantage that for each sample kinetic data was obtained.

We also evaluated the diffraction patterns obtained in the presence of the antifreeze-active compounds noting the loss of intensity of the (002) peak. This could be interpreted as indicating specific ice binding, but we show with controls that this cannot be inferred due to preferred orientations and insufficient crystallites within the scattering volume. This confirms that the method is a useful addition to the suite of tools for discovering and investigating new ice recrystallization inhibitors but that, in the set up used here, care must be taken not to overfit the data and extrapolate information about specific crystal face binding.

\section{Conflicts of interest}

There are no conflicts to declare.

\section{Acknowledgements}

M.I.G. holds an ERC starting grant (CRYOMAT 638661), and is a Royal Society Industry Fellow (R1 $\backslash 191037)$. The Royal Society are also thanked for funding the cryo-microscopes used in this study. We would also like to thank Peter Davies (Queen's University, Kingston, Canada) for providing the genetic construct encoding for AFPIII from ocean pout (rQAE isoform, M1.1HISPET20b), and A. L. DeVries (University of Illinois at Urbana-Champaign, USA) for kindly providing the AFGP8 used in this work. We thank the University of Warwick X-ray Diffraction Research Technology Platform for the XRD measurements.

\section{References}

1 A. J. Scotter, C. B. Marshall, L. A. Graham, J. A. Gilbert, C. P. Garnham and P. L. Davies, The Basis for Hyperactivity of Antifreeze Proteins, Cryobiology, 2006, 53(2), 229239.

2 M. Mangiagalli, M. Bar-Dolev, P. Tedesco, A. Natalello, A. Kaleda, S. Brocca, D. de Pascale, S. Pucciarelli, C. Miceli, I. Bravslavsky, et al. Cryo-Protective Effect of an Ice-Binding Protein Derived from Antarctic Bacteria, FEBS J., 2016, 284, 163-177. 
3 C. J. Capicciotti, M. Leclere, F. A. Perras, D. L. Bryce, H. Paulin, J. Harden, Y. Liu and R. N. Ben, Potent Inhibition of Ice Recrystallization by Low Molecular Weight Carbohydrate-Based Surfactants and Hydrogelators, Chem. Sci., 2012, 3(5), 1408-1416.

4 M. Smallwood, D. Worrall, L. Byass, L. Elias, D. Ashford, C. J. Doucet, C. Holt, J. Telford, P. Lillford and D. J. Bowles, Isolation and Characterization of a Novel Antifreeze Protein from Carrot (Daucus Carota), Biochem. J., 1999, 340, 385391.

5 D. Nichols, J. Bowman, K. Sanderson, C. M. Nichols, T. Lewis, T. McMeekin and P. D. Nichols, Developments with Antarctic Microorganisms: Culture Collections, Bioactivity Screening, Taxonomy, PUFA Production and Cold-Adapted Enzymes, Curr. Opin. Biotechnol., 1999, 10(3), 240-246.

6 J. R. Layne and A. L. Jones, Freeze Tolerance in the Gray Treefrog: Cryoprotectant Mobilization and Organ Dehydration, J. Exp. Zool., 2001, 290(1), 1-5.

7 X. Wen, S. Wang, J. G. Duman, J. F. Arifin, V. Juwita, W. A. Goddard, A. Rios, F. Liu, S.-K. Kim, R. Abrol, et al. Antifreeze Proteins Govern the Precipitation of Trehalose in a Freezing-Avoiding Insect at Low Temperature, Proc. Natl. Acad. Sci. U. S. A., 2016, 113(24), 6683-6688.

8 A. K. Balcerzak, C. J. Capicciotti, J. G. Briard and R. N. Ben, Designing Ice Recrystallization Inhibitors : From Antifreeze (Glyco) Proteins to Small Molecules, RSC Adv., 2014, 4, 42682-42696, DOI: 10.1039/c4ra06893a.

9 M. M. Harding, P. I. Anderberg and A. D. J. Haymet, "Antifreeze" Glycoproteins from Polar Fish, Eur. J. Biochem., 2003, 270(7), 1381-1392.

10 P. L. Davies, Ice-Binding Proteins: A Remarkable Diversity of Structures for Stopping and Starting Ice Growth, Trends Biochem. Sci., 2014, 39(11), 548-555.

11 P. Mazur, Cryobiology: The Freezing of Biological Systems, Science, 1970, 168(3934), 939-949.

12 C. I. Biggs, T. L. Bailey, B. Graham, C. Stubbs, A. E. R. Fayter and M. I. Gibson, Polymer Mimics of Biomacromolecular Antifreezes, Nat. Commun., 2017, 8(1), 1546, DOI: 10.1038/ s41467-017-01421-7.

13 M. I. Gibson, Slowing the Growth of Ice with Synthetic Macromolecules: Beyond Antifreeze (Glyco)Proteins, Polym. Chem., 2010, 1(8), 1141-1152.

14 B. Graham, A. E. R. Fayter, J. E. Houston, R. C. Evans and M. I. Gibson, Facially Amphipathic Glycopolymers Inhibit Ice Recrystallization, J. Am. Chem. Soc., 2018, 140(17), 5682-5685.

15 C. I. Biggs, C. Stubbs, B. Graham, A. E. R. Fayter, M. Hasan and M. I. Gibson, Mimicking the Ice Recrystallization Activity of Biological Antifreezes. When Is a New Polymer "Active"?, Macromol. Biosci., 2019, 19(7), 1-9.

16 S. Liu and R. N. Ben, C-Linked, Galactosyl Serine AFGP Analogues as Potent Recrystallization Inhibitors, Org. Lett., 2005, 7(12), 2385-2388.

17 R. N. Ben, A. A. Eniade and L. Hauer, Synthesis of a C-Linked Antifreeze Glycoprotein (AFGP) Mimic: Probes for
Investigating the Mechanism of Action, Org. Lett., 1999, 1(11), 1759-1762.

18 M. Leclère, B. K. Kwok, L. K. Wu, D. S. Allan and R. N. Ben, C-Linked Antifreeze Glycoprotein (C -AFGP) Analogues as Novel Cryoprotectants, Bioconjugate Chem., 2011, 22(9), 1804-1810.

19 C. J. Capicciotti, J. D. R. R. Kurach, T. R. Turner, R. S. Mancini, J. P. Acker and R. N. Ben, Small Molecule Ice Recrystallization Inhibitors Enable Freezing of Human Red Blood Cells with Reduced Glycerol Concentrations, Sci. Rep., 2015, 5(9692), 1-10, DOI: 10.1038/srep09692.

20 J. G. Briard, S. Jahan, P. Chandran, D. Allan, N. Pineault and R. N. Ben, Small-Molecule Ice Recrystallization Inhibitors Improve the Post-Thaw Function of Hematopoietic Stem and Progenitor Cells, ACS Omega, 2016, 1(5), 1010-1018, DOI: 10.1021/acsomega.6b00178.

21 C. Stubbs, J. Lipecki and M. I. Gibson, Regioregular Alternating Polyampholytes Have Enhanced Biomimetic Ice Recrystallization Activity Compared to Random Copolymers and the Role of Side Chain versus Main Chain Hydrophobicity, Biomacromolecules, 2017, 18(1), 295-302, DOI: 10.1021/acs.biomac.6b01691.

22 R. Rajan, F. Hayashi, T. Nagashima and K. Matsumura, Toward a Molecular Understanding of the Mechanism of Cryopreservation by Polyampholytes: Cell Membrane Interactions and Hydrophobicity, Biomacromolecules, 2016, 17(5), 1882-1893, DOI: 10.1021/acs.biomac.6b00343.

23 G. Bai, Z. Song, H. Geng, D. Gao, K. Liu, S. Wu, W. Rao, L. Guo and J. Wang, Oxidized Quasi-Carbon Nitride Quantum Dots Inhibit Ice Growth, Adv. Mater., 2017, 29(28), 1-8, DOI: 10.1002/adma.201606843.

24 R. Drori, C. Li, C. Hu, P. Raiteri, A. L. Rohl, M. D. Ward and B. Kahr, A Supramolecular Ice Growth Inhibitor, J. Am. Chem. Soc., 2016, 138(40), 13396-13401, DOI: 10.1021/ jacs.6b08267.

25 H. Geng, X. Liu, G. Shi, G. Bai, J. Ma, J. Chen, Z. Wu, Y. Song, H. Fang and J. Wang, Graphene Oxide Restricts Growth and Recrystallization of Ice Crystals, Angew. Chem., Int. Ed., 2017, 56(4), 997-1001, DOI: 10.1002/ anie.201609230.

26 O. Mizrahy, M. Bar-Dolev, S. Guy and I. Braslavsky, Inhibition of Ice Growth and Recrystallization by Zirconium Acetate and Zirconium Acetate Hydroxide, PLoS One, 2013, 8(3), e59540, DOI: 10.1371/journal. pone.0059540.

27 T. Li, Y. Zhao, Q. Zhong and T. Wu, Inhibiting Ice Recrystallization by Nanocelluloses, Biomacromolecules, 2019, 20(4), 1667-1674, DOI: 10.1021/acs.biomac.9b00027.

28 T. Congdon, R. Notman and M. I. Gibson, Antifreeze (Glyco)Protein Mimetic Behavior of Poly(Vinyl Alcohol): Detailed Structure Ice Recrystallization Inhibition Activity Study, Biomacromolecules, 2013, 14(5), 1578-1586, DOI: 10.1021/bm400217j.

29 N. S. Vail, C. Stubbs, C. I. Biggs and M. I. Gibson, Ultralow Dispersity Poly(Vinyl Alcohol) Reveals Significant Dispersity Effects on Ice Recrystallization Inhibition 
Activity, ACS Macro Lett., 2017, 6(9), 1001-1004, DOI: 10.1021/acsmacrolett.7b00595.

30 T. Inada and S. Lu, Inhibition of Recrystallization of Ice Grains by Adsorption of Poly(Vinyl Alcohol) onto Ice Surfaces, Cryst. Growth Des., 2003, 3(5), 747-752, DOI: 10.1021/cg0340300.

31 T. R. Congdon, R. Notman and M. I. Gibson, Synthesis of Star-Branched Poly(Vinyl Alcohol) and Ice Recrystallization Inhibition Activity, Eur. Polym. J., 2017, 88, 320-327, DOI: 10.1016/j.eurpolymj.2017.01.039.

32 C. A. Knight, J. Hallett and A. L. DeVries, Solute Effects on Ice Recrystallization: An Assessment Technique, Cryobiology, 1988, 25, 55-60, DOI: 10.1016/0011-2240(88)90020-X.

33 C. Budke and T. Koop, Ice Recrystallization Inhibition and Molecular Recognition of Ice Faces by Poly(Vinyl Alcohol), ChemPhysChem, 2006, 7(12), 2601-2606, DOI: 10.1002/ cphc.200600533.

34 M. A. Graewert and D. I. Svergun, Impact and Progress in Small and Wide Angle X-Ray Scattering (SAXS and WAXS), Curr. Opin. Struct. Biol., 2013, 23(5), 748-754, DOI: 10.1016/ j.sbi.2013.06.007.

35 K. D. M. Harris, M. Tremayne and B. M. Kariuki, Contemporary Advances in the Use of Powder X-Ray Diffraction for Structure Determination, Angew. Chem., Int. Ed., 2001, 40, 1626-1651, DOI: 10.1002/chin.200132293.

36 C. Giacovazzo, Fundamentals of Crystallography, International Union of Crystallography, Oxford, 1992.

37 X. Chen, J. Schröder, S. Hauschild, S. Rosenfeldt, M. Dulle and S. Förster, Simultaneous SAXS/WAXS/UV-Vis Study of the Nucleation and Growth of Nanoparticles: A Test of Classical Nucleation Theory, Langmuir, 2015, 31(42), 11678-11691, DOI: 10.1021/acs.langmuir.5b02759.

38 J. C. Houle, A. V. Chen, A. C. Brenna, K. L. Mealey and A. M. Kiszonas, Determination of Optimal Storage Temperature and Duration for Analysis of Total and Isoenzyme Lactate Dehydrogenase Activities in Canine Serum and Cerebrospinal Fluid, Vet. Clin. Pathol., 2015, 44(2), 253-261, DOI: 10.1111/vcp.12263.

39 D. R. Hummer, P. J. Heaney and J. E. Post, In Situ Observations of Particle Size Evolution during the Hydrothermal Crystallization of TiO2: A Time-Resolved Synchrotron SAXS and WAXS Study, J. Cryst. Growth, 2012, 344(1), 51-58, DOI: 10.1016/j.jcrysgro.2012.01.044.

40 R. N. Andrews, J. Serio, G. Muralidharan and J. Ilavsky, An in Situ USAXS-SAXS-WAXS Study of Precipitate Size Distribution Evolution in a Model Ni-Based Alloy, J. Appl. Crystallogr., 2017, 50(3), 734-740, DOI: 10.1107/ S1600576717006446.

41 W. H. Bragg, The Crystal Structure of Ice, Proc. Phys. Soc., 1922, 34, 98-103.

42 C. G. Salzmann, P. G. Radaelli, B. Slater, J. L. Finney and J. L. Finney, The Polymorphism of Ice: Five Unresolved Questions, Phys. Chem. Chem. Phys., 2011, 13(41), 1846818480, DOI: 10.1039/c1cp21712g.

43 L. L. C. Olijve, T. J. Sun, T. Narayanan, C. Jud, P. L. Davies and I. K. Voets, Solution Structure of Hyperactive Type I
Antifreeze Protein, RSC Adv., 2013, 3(17), 5903-5908, DOI: 10.1039/c3ra22729d.

44 J. Michielsen, J. Dings and J. van der Elsken, Small-Angle $\mathrm{x}$-Ray Scattering in the Early Stages of Ice Formation, Phys. Rev. A, 1991, 44(6), 4068-4071.

45 E. J. Shalaev, D. V. Malakhov, A. N. Kanev, V. I. Kosyakov, F. V. Tuzikov, N. A. Varaksin and V. I. Vavilin, Study of the Phase Diagram Water Fraction of the System WaterGlycine-Sucrose by DTA and X-Ray Diffraction Methods, Thermochim. Acta, 1992, 196(1), 213-220, DOI: 10.1016/ 0040-6031(92)85021-M.

46 B. Zakharov, A. Fisyuk, A. Fitch, Y. Watier, A. Kostyuchenko, D. Varshney, M. Sztucki, E. Boldyreva and E. Shalaev, Ice Recrystallization in a Solution of a Cryoprotector and Its Inhibition by a Protein: Synchrotron X-Ray Diffraction Study, J. Pharm. Sci., 2016, 105(7), 21292138, DOI: 10.1016/j.xphs.2016.04.020.

47 D. B. Varshney, J. A. Elliott, L. A. Gatlin, S. Kumar, R. Suryanarayanan and E. Y. Shalaev, Synchrotron X-Ray Diffraction Investigation of the Anomalous Behavior of Ice During Freezing of Aqueous Systems, J. Phys. Chem. B, 2009, 113(18), 6177-6182, DOI: 10.1021/jp900404m.

48 L. E. Wilkins, M. Hasan, A. E. R. Fayter, C. Biggs, M. Walker and M. I. Gibson, Site-Specific Conjugation of Antifreeze Proteins onto Polymer-Stabilized Nanoparticles, Polym. Chem., 2019, 10, 2986-2990, DOI: 10.1039/ c8py01719k.

49 C. A. Knight, D. Wen and R. A. Laursen, Nonequilibrium Antifreeze Peptides and the Recrystallization of Ice, Cryobiology, 1995, 32(1), 23-34, DOI: 10.1006/ cryo.1995.1002.

50 J. P. Pezacki, M. Noestheden, R. N. Ben, J. Jackman, D. Moffat and S. Findlay, Assessing Antifreeze Activity of AFGP 8 Using Domain Recognition Software, Biochem. Biophys. Res. Commun., 2007, 354(2), 340-344, DOI: 10.1016/j.bbrc.2006.12.225.

51 C. Budke, C. Heggemann, M. Koch, N. Sewald and T. Koop, Ice Recrystallization Kinetics in the Presence of Synthetic Antifreeze Glycoprotein Analogues Using the Framework of LSW Theory, J. Phys. Chem. B, 2009, 113(9), 2865-2873, DOI: $10.1021 /$ jp805726e.

52 S. Wang, N. Amornwittawat, J. Banatlao, M. Chung, Y. Kao and X. Wen, Hofmeister Effects of Common Monovalent Salts on the Beetle Antifreeze Protein Activity, J. Phys. Chem. B, 2009, 113(42), 13891-13894, DOI: 10.1021/ jp907762u.

53 R. Surís-Valls and I. K. Voets, The Impact of Salts on the Ice Recrystallization Inhibition Activity of Antifreeze (Glyco) Proteins, Biomolecules, 2019, 9(8), 347.

54 T. L. Malkin, B. J. Murray, V. Andrey, J. Anwar, C. G. Salzmann, T. L. Malkin, B. J. Murray, A. V. Brukhno, J. Anwar and C. G. Salzmann, Structure of Ice Crystallized from Supercooled Water, Proc. Natl. Acad. Sci. U. S. A., 2012, 109(4), 1041-1045, DOI: 10.1073/pnas.1201020109.

55 Y. E. Yagci, M. Antonietti and H. G. Börner, Synthesis of Poly(Tartar Amides) as Bio-Inspired Antifreeze Additives, 
Macromol. Rapid Commun., 2006, 27(19), 1660-1664, DOI: 10.1002/marc.200600451.

56 E. Baruch and Y. Mastai, Antifreeze Properties of Polyglycidol Block Copolymers, Macromol. Rapid Commun., 2007, 28(23), 2256-2261, DOI: 10.1002/marc.200700501.
57 S. Deville, C. Viazzi, J. Leloup, A. Lasalle, C. Guizard, E. Maire, J. Adrien and L. Gremillard, Ice Shaping Properties, Similar to That of Antifreeze Proteins, of a Zirconium Acetate Complex, PLoS One, 2011, 6(10), e26474. 